hep-th/0312216

December 2003

\title{
Adventures in Thermal Duality (I): Extracting Closed-Form Solutions for Finite-Temperature Effective Potentials in String Theory
}

\author{
Keith R. Dienes* and Michael Lennek ${ }^{\dagger}$ \\ Department of Physics, University of Arizona, Tucson, AZ 85721 USA
}

\begin{abstract}
Thermal duality, which relates the physics of closed strings at temperature $T$ to the physics at the inverse temperature $1 / T$, is one of the most intriguing features of string thermodynamics. Unfortunately, the classical definitions of thermodynamic quantities such as entropy and specific heat are not invariant under the thermal duality symmetry. In this paper, we investigate whether there might nevertheless exist special solutions for the string effective potential such that the duality symmetry will be preserved for all thermodynamic quantities. Imposing this as a constraint, we derive a series of unique functional forms for the complete temperature-dependence of the required string effective potentials. Moreover, we demonstrate that these solutions successfully capture the leading temperature behavior of a variety of actual one-loop effective potentials for duality-covariant finite-temperature string ground states. This leads us to conjecture that our solutions might actually represent the exact effective potentials when contributions from all orders of perturbation theory are included.
\end{abstract}

\footnotetext{
*E-mail address: dienes@physics.arizona.edu

${ }^{\dagger}$ E-mail address: mlennek@physics.arizona.edu
} 


\section{Introduction}

Some of the most intriguing features of string theory have been the existence of numerous dualities which connect physics in what would otherwise appear to be vastly dissimilar regimes. Such dualities include strong/weak coupling duality (S-duality) as well as large/small compactification radius duality (T-duality), and together these form the bedrock upon which much of our understanding of the full, non-perturbative moduli space of string theory is based.

There is, however, an additional duality which has received far less scrutiny: this is thermal duality, which relates string theory at temperature $T$ with string theory at the inverse temperature $T_{c}^{2} / T$ where $T_{c}$ is a critical (or self-dual) temperature related to the string scale. Thermal duality follows naturally from T-duality and Lorentz invariance, and thus has roots which are as deep as the dualities that occur at zero temperature. Given the importance of dualities of all sorts in extending our understanding of the unique features of non-perturbative string theory, we are led to ask what new insights can be gleaned from a study of thermal duality.

In this paper, we shall focus on the first feature that immediately strikes any student of this subject: classical thermodynamics, as currently formulated, is not invariant (or covariant) under thermal duality. While certain thermodynamic quantities such as the free energy and the internal energy of an ideal closed string gas exhibit invariances (or covariances) under thermal duality transformations, other quantities such as entropy and specific heat do not.

In this paper, we shall investigate whether thermal duality might nevertheless happen to be preserved for special choices of the effective potential. In other words, we shall investigate whether it is possible to construct an effective potential such that all corresponding physically relevant thermodynamic quantities will turn out to be duality covariant. Thus, in this way, we seek to exploit thermal duality in order to constrain the effective potential in a manner that transcends a direct order-by-order perturbative calculation.

Remarkably, we shall find that there exist a unique series of functional forms which have this property. Moreover, we shall demonstrate that these solutions successfully capture the leading temperature dependence of the one-loop effective potentials for a variety of finite-temperature string ground states involving time/temperature compactifications on $S^{1}$ (circles) and $S^{1} / Z_{2}$ (orbifolds) in all dimensions $D \geq 2$. The precision with which this occurs leads us to conjecture that our solutions might actually represent the exact solutions for the corresponding string effective potentials when results from all orders of perturbation theory are included.

Note that a preliminary summary of some of these results has appeared in Ref. [1]. Our goal here is to provide a more complete and self-contained discussion and derivation of these results. There are, however, numerous topics pertaining to string thermodynamics which we will not address in this paper. These include the nature of the Hagedorn phase transition as well as the Jeans instability and general issues concern- 
ing the interplay between gravity and thermodynamics. It would be interesting to explore the extent to our results concerning thermal duality can shed light on these issues, and we hope to address these questions in future work.

\section{Thermal duality and the rules of thermodynamics}

Let us begin by quickly presenting some of the key ideas that will be relevant for our discussion. Our goal will be to highlight the manner in which the rules of standard thermodynamics generally tend to break thermal duality.

Just as in ordinary statistical mechanics, the fundamental quantity of interest in string thermodynamics is the one-loop thermal string partition function $Z_{\text {string }}(\tau, T)$. This partition function generally exhibits the symmetries of the underlying theory. For example, we shall assume that $Z_{\text {string }}$ is invariant under modular transformations:

$$
Z_{\text {string }}(\tau+1, T)=Z_{\text {string }}(-1 / \tau, T)=Z_{\text {string }}(\tau, T)
$$

where $\tau$ is the complex modular parameter describing the shape of the one-loop (toroidal) worldsheet. Modular invariance is required for the consistency of the corresponding closed string model, and arises from the assumption of conformal invariance at the one-loop level.

More importantly, however, we shall also assume that $Z_{\text {string }}$ is invariant under thermal duality:

$$
Z_{\text {string }}\left(\tau, T_{c}^{2} / T\right)=Z_{\text {string }}(\tau, T)
$$

where $T_{c}$ is the self-dual temperature. Thermal duality also has deep roots (for early papers, see Refs. [2, 3, 4, 5, 6, 7]). In general, finite-temperature effects can be incorporated into string theory [8] by compactifying an additional time dimension on a circle (or orbifold [9]) of radius $R_{T}=(2 \pi T)^{-1}$. However, Lorentz invariance guarantees that the properties of this extra time dimension should be the same as those of the original space dimensions, and T-duality [10, 11, 12, tells us that closed string theory on a compactified space dimension of radius $R$ is indistinguishable from that on a space of radius $R_{c}^{2} / R$ where $R_{c}=\sqrt{\alpha^{\prime}}$ is the self-dual radius. Together, these symmetries then imply thermal duality, with $T_{c} \equiv M_{\text {string }} / 2 \pi$. Note that the thermal duality symmetry holds to all orders in perturbation theory [5].

All thermodynamic quantities of interest are generated from $Z_{\text {string }}$. The finitetemperature vacuum amplitude $\mathcal{V}(T)$ is given by [8, 13, 2]

$$
\mathcal{V}(T) \equiv-\frac{1}{2} \mathcal{M}^{D-1} \int_{\mathcal{F}} \frac{d^{2} \tau}{(\operatorname{Im} \tau)^{2}} Z_{\text {string }}(\tau, T)
$$

where $\mathcal{M} \equiv M_{\text {string }} / 2 \pi$ is the reduced string scale; $D$ is the number of non-compact spacetime dimensions; and $\mathcal{F} \equiv\left\{\tau:|\operatorname{Re} \tau| \leq \frac{1}{2}, \operatorname{Im} \tau>0,|\tau| \geq 1\right\}$ is the fundamental domain of the modular group. Note that $T_{c}=\mathcal{M}$. In general, $\mathcal{V}(T)$ plays the 
role usually taken by the logarithm of the statistical-mechanical partition function. Because of its role in governing the dynamics of the theory, we shall occasionally refer to the vacuum amplitude $\mathcal{V}(T)$ as the "effective potential" even though this terminology is often used instead to describe the free energy $F$. Given this definition for $\mathcal{V}$, the free energy $F$, internal energy $U$, entropy $S$, and specific heat $c_{V}$ then follow from the standard thermodynamic definitions:

$$
F=T \mathcal{V}, \quad U=-T^{2} \frac{d}{d T} \mathcal{V}, \quad S=-\frac{d}{d T} F, \quad c_{V}=\frac{d}{d T} U
$$

It is easy to see that the thermal duality invariance of $Z_{\text {string }}$ is inherited by some of its descendants. Since $\mathcal{V}$ is just the modular integral of $Z_{\text {string }}, \mathcal{V}$ is also invariant under thermal duality transformations:

$$
\mathcal{V}\left(T_{c}^{2} / T\right)=\mathcal{V}(T)
$$

Likewise, it is easy to verify that the free energy $F$ and the internal energy $U$ transform covariantly under thermal duality:

$$
F\left(T_{c}^{2} / T\right)=\left(\frac{T_{c}}{T}\right)^{2} F(T), \quad U\left(T_{c}^{2} / T\right)=-\left(\frac{T_{c}}{T}\right)^{2} U(T) .
$$

Thus, these quantities also respect the thermal duality symmetry; in fact, this symmetry sets a zero for the internal energy such that $U\left(T_{c}\right)=0$.

Unfortunately, the entropy and specific heat fail to have any closed transformation properties under the thermal duality symmetry. Specifically, we find

$$
\begin{aligned}
S\left(T_{c}^{2} / T\right) & =-S(T)-2 F(T) / T, \\
c_{V}\left(T_{c}^{2} / T\right) & =c_{V}(T)-2 U(T) / T .
\end{aligned}
$$

This failure to transform covariantly suggests that entropy and specific heat are improperly defined from a string-theoretic standpoint. At best, they are not the proper "eigenquantities" which should correspond to physical observables.

It is easy to diagnose the source of this problem. In general, a function $f(T)$ will be called thermal duality covariant with weight $k$ and sign $\gamma= \pm 1$ if, under the thermal duality transformation $T \rightarrow T_{c}^{2} / T$, we find

$$
f(T) \rightarrow f\left(T_{c}^{2} / T\right)=\gamma\left(T_{c} / T\right)^{k} f(T)
$$

Thus, $\mathcal{V}$ has $(k, \gamma)=(0,1)$, while $F$ and $U$ have $(k, \gamma)=(2,1)$ and $(2,-1)$ respectively. Note that $\gamma= \pm 1$ are the only two possible choices consistent with the $\mathbb{Z}_{2}$ nature of the thermal duality transformation. In general, multiplication by $T$ is a covariant operation, resulting in a function with weight $k+2$ and the same sign for $\gamma$. However, the temperature derivative $d / d T$ generally breaks duality covariance. 
To see this, let us imagine that $f(T)$ has weight $k$ and sign $\gamma$. Evaluating $d f / d T$ at temperature $T_{c}^{2} / T$, we then find

$$
\begin{aligned}
{\left[\frac{d f}{d T}\right]\left(T_{c}^{2} / T\right) } & =\frac{d}{d\left(T_{c}^{2} / T\right)} f\left(T_{c}^{2} / T\right) \\
& =-\gamma\left(\frac{T}{T_{c}}\right)^{2} \frac{d}{d T}\left[\left(T_{c} / T\right)^{k} f(T)\right] \\
& =-\gamma\left(\frac{T_{c}}{T}\right)^{k-2}\left(\frac{d f}{d T}-\frac{k f}{T}\right) .
\end{aligned}
$$

Thus, as a result of the second term above, we see that $d f / d T$ fails to transform covariantly under the thermal duality transformation unless $f$ itself has $k=0$. Since the vacuum amplitude $\mathcal{V}$ has $k=0$, this explains why the internal energy $U$ continues to be duality covariant (with $k=2$ ) even though it involves a temperature derivative. However, since the free energy $F$ and the internal energy $U$ each already have $k=2$, we see that subsequent derivatives yield quantities (such as the entropy $S$ and specific heat $c_{V}$ ) which are no longer duality covariant.

\section{Special solutions for string effective potentials}

Let us now consider whether there might exist special finite-temperature vacuum amplitudes $\mathcal{V}(T)$ in which thermal duality covariance is preserved for all thermodynamic quantities. In other words, we shall seek special solutions for $\mathcal{V}(T)$ such that all of its thermodynamic descendants turn out to be duality covariant, even though the rules by which these quantities are calculated explicitly break this symmetry. We emphasize that in choosing this line of attack, we are necessarily losing generality; we are essentially limiting our attention to special, highly symmetric string ground states. Nevertheless, as we shall see, it is important to investigate this possibility.

\subsection{General approach}

In order to proceed along these lines, we first need to address a general mathematical question: from amongst all duality-covariant functions $f(T)$ of weight $k$ and sign $\gamma$, are there any special functions $f(T)$ for which $d f / d T$ "accidentally" turns out to be covariant?

Given the derivative in Eq. (2.9), we see that there is only one way in which $d f / d T$ can possibly be thermal duality covariant: we must have

$$
\frac{d f}{d T}-\frac{k f(T)}{T}=-\delta\left(\frac{T_{c}}{T}\right)^{\ell} \frac{d f}{d T}
$$

for some $\operatorname{sign} \delta$ and exponent $\ell$. If Eq. (3.1) is satisfied, then we see from Eq. (2.9) that $d f / d T$ will indeed be covariant, with sign $\gamma \delta$ and weight $k+\ell-2$. Note that we 
must have $\delta= \pm 1$ in order to produce a consistent sign for $d f / d T$. (The minus sign in front of $\delta$ has been inserted for future convenience.)

It is not difficult to find solutions for $f(T)$ in Eq. (3.1), since this is nothing but a linear first-order differential equation. For $\ell \neq 0$, we thus obtain the general solution

$$
f \sim\left(T^{\ell}+\delta T_{c}^{\ell}\right)^{k / \ell}
$$

where we are disregarding an overall, arbitrary, $T$-independent normalization factor. However, in this derivation we assumed that $f$ has weight $k$ and sign $\gamma$. Checking the solution in Eq. (3.2), we find that this does not restrict the value of $\ell$, but does require that $\delta^{k / \ell}=\gamma$.

By contrast, if $\ell=0$ in Eq. (3.1), we obtain a non-zero solution for $f(T)$ only if $\gamma=1$ and $\delta=+1$ :

$$
f \sim T^{k / 2} .
$$

As required, this also has weight $k$.

Thus, from amongst all possible covariant functions $f(T)$ with weight $k$ and sign $\gamma$, we have found that only an extremely restrictive form for $f(T)$ guarantees that $d f / d T$ is also thermal duality covariant: either $f(T)$ must have the form given in Eq. (3.2) where $\ell \neq 0$ is arbitrary and where $\delta^{k / \ell}=\gamma$, with $\delta= \pm 1$; or $f(T)$ must have the form given in Eq. (3.3), which can occur only if $\gamma=1$. Of course, overall multiplicative factors of $T_{c}$ can always be introduced in either expression as needed on dimensional grounds.

\subsection{Preserving duality covariance for entropy and specific heat: A thermal duality "bootstrap"}

Using this, let us now reconsider our original thermodynamic problem. We begin with a vacuum amplitude $\mathcal{V}$, which we assume invariant under thermal duality transformations. Thus, $\mathcal{V}$ necessarily has $k=0$ and $\gamma=1$. From this, we proceed to derive $F$ and $U$. Once again, these quantities are also automatically duality covariant; they each have weight $k=2$ and their signs are $\gamma=+1$ and -1 respectively. Up to this point, the functional forms for $\mathcal{V}, F$, and $U$ are completely arbitrary (subject to the above constraints on their weights and signs). However, it is in calculating $S$ and $c_{V}$ that potential difficulties arise, for we must demand that $S$ and $c_{V}$ be simultaneously covariant as well. This then provides two new non-trivial constraints on the forms of $F$ and $U$, as discussed above. Working backwards, this then provides a very restrictive set of possibilities for the vacuum amplitudes $\mathcal{V}$ from which both $F$ and $U$ are derived. In other words, we will have essentially used a "bootstrap" formed by demanding the covariance of $S$ and $c_{V}$ to deduce a particular form (or set of forms) of the vacuum amplitude $\mathcal{V}$.

Carrying out this calculation is relatively straightforward. We first focus on the entropy $S$. In order for $S$ to be thermal duality covariant, the free energy $F$ (which 
must have weight $k=2$ and $\operatorname{sign} \gamma=1$ ) is required to take the form

$$
F(T) \sim-\frac{\left(T^{\ell}+\delta T_{c}^{\ell}\right)^{2 / \ell}}{T_{c}}
$$

where

$$
\delta^{2 / \ell}=1
$$

Note that the factor of $T_{c}$ in the denominator of Eq. (3.4) has been inserted on dimensional grounds (where we implicitly express our thermodynamic quantities in units of $\mathcal{M}^{D-1}$ ); likewise, we have also inserted an overall minus sign for future convenience. Also note that Eq. (3.5) restricts us to $\delta=+1$ for even $\ell$, but allows $\delta= \pm 1$ for odd $\ell$. This form for $F$ guarantees that $S$, which takes the form

$$
S(T) \sim 2 \frac{T^{\ell-1}}{T_{c}}\left(T^{\ell}+\delta T_{c}^{\ell}\right)^{2 / \ell-1},
$$

is covariant with weight $\ell$ and $\operatorname{sign} \delta$.

We are of course deliberately disregarding the $\ell=0$ possibility, stemming from Eq. (3.3), that $F(T) \sim T$. We reject this possibility not only because this would make $F(T)$ independent of $T_{c}$ (which is unexpected from a string calculation), but also because it leads to an entropy which is completely temperature-independent and hence unphysical.

Given $F(T)$ in Eq. (3.4), we immediately determine that $\mathcal{V}(T)$ must take the general form

$$
\mathcal{V}(T) \sim-\frac{\left(T^{\ell}+\delta T_{c}^{\ell}\right)^{2 / \ell}}{T T_{c}} .
$$

Note that this is indeed invariant under thermal duality transformations, as required. This in turn implies that $U(T)$ must have the general form

$$
U(T) \sim \frac{1}{T_{c}}\left(T^{\ell}+\delta T_{c}^{\ell}\right)^{2 / \ell-1}\left(T^{\ell}-\delta T_{c}^{\ell}\right)
$$

which is of course consistent with our requirement that $U$ have weight 2 and sign -1 . Thus, up to this point, we have found that the entropy will be thermal duality covariant (along with the effective potential, the free energy, and the internal energy) if and only if $\mathcal{V}(T)$ takes the form (3.7).

We now impose our requirement that $c_{V}$ also be thermal duality covariant. As we shall see, this will provide a constraint on the value of $\ell$. Since $U(T)$ is given in Eq. (3.8), we can immediately calculate the specific heat, obtaining

$$
c_{V}(T) \sim 2 \frac{T^{\ell-1}}{T_{c}}\left(T^{\ell}+\delta T_{c}^{\ell}\right)^{2 / \ell-2}\left[T^{\ell}+(\ell-1) \delta T_{c}^{\ell}\right] .
$$

Clearly, this quantity fails to be duality covariant unless the final factor in square brackets takes the form $T^{\ell} \pm \delta T_{c}^{\ell}$ with $\delta= \pm 1$, or unless this factor takes the form $T^{\ell}$ 
(in which case this factor joins with the overall $T^{\ell-1}$ prefactor to modify the duality weight of $c_{V}$ ). These two options occur only for $\ell=2$ or $\ell=1$ respectively.

Note that the $\ell=1,2$ cases provide maximal duality symmetry for our solutions. Indeed, in these cases, our solution for $U(T)$ also simultaneously takes the form

$$
U(T) \sim \frac{\left(T^{m}+\epsilon T_{c}^{m}\right)^{2 / m}}{T_{c}}
$$

for some $m$ and sign $\epsilon= \pm 1$ (with $\epsilon^{2 / m}=-1$ ), as required from Eq. (3.2) in order to yield a covariant specific heat $c_{V}=d U / d T$. Moreover, since the specific heat is also given by the relation $c_{V}=T d S / d T$, our solution for $S(T)$ also takes this same special form in these cases.

Thus, summarizing, we see that our requirement of preserving general covariance for our thermodynamic quantities forces them to have a particular form:

$$
\begin{aligned}
\mathcal{V}^{(\ell)}(T) & \sim-\left(T^{\ell}+\delta T_{c}^{\ell}\right)^{2 / \ell} / T T_{c} \\
F^{(\ell)}(T) & \sim-\left(T^{\ell}+\delta T_{c}^{\ell}\right)^{2 / \ell} / T_{c} \\
U^{(\ell)}(T) & \sim\left(T^{\ell}+\delta T_{c}^{\ell}\right)^{2 / \ell-1}\left(T^{\ell}-\delta T_{c}^{\ell}\right) / T_{c} \\
S^{(\ell)}(T) & \sim 2 T^{\ell-1}\left(T^{\ell}+\delta T_{c}^{\ell}\right)^{2 / \ell-1} / T_{c} \\
c_{V}^{(\ell)}(T) & \sim 2 T^{\ell-1}\left(T^{\ell}+\delta T_{c}^{\ell}\right)^{2 / \ell-2}\left[T^{\ell}+(\ell-1) \delta T_{c}^{\ell}\right] / T_{c}
\end{aligned}
$$

where

$$
\delta= \begin{cases}+1 & \ell \text { even } \\ \pm 1 & \ell \text { odd }\end{cases}
$$

These solutions are plotted in Fig. [1, and ensure that $\mathcal{V}, F, U$, and $S$ are all thermal duality covariant for any value of $\ell$. While $\mathcal{V}, F$, and $U$ have duality weights $(k, \gamma)=$ $(0,1),(2,1)$, and $(2,-1)$ respectively, the entropy $S$ has duality weight and sign $(k, \gamma)=(\ell, \delta)$. Observe that the traditional relation $U=F+T S$ continues to hold for all $\ell$.

However, $c_{V}$ will also be thermal duality covariant if and only if $\ell=1$ or $\ell=2$. The explicit solutions in these cases reduce to

$$
\begin{aligned}
\ell=2: \quad \mathcal{V}^{(2)}(T) & =-\left(T^{2}+T_{c}^{2}\right) /\left(T T_{c}\right) \\
F^{(2)}(T) & =-\left(T^{2}+T_{c}^{2}\right) / T_{c} \\
U^{(2)}(T) & =\left(T^{2}-T_{c}^{2}\right) / T_{c} \\
S^{(2)}(T) & =2 T / T_{c} \\
c_{V}^{(2)}(T) & =2 T / T_{c}
\end{aligned}
$$

and

$$
\begin{array}{ll}
\ell=1: \quad \mathcal{V}^{(1)}(T)=-\left(T+\delta T_{c}\right)^{2} /\left(T T_{c}\right) \\
F^{(1)}(T)=-\left(T+\delta T_{c}\right)^{2} / T_{c}
\end{array}
$$



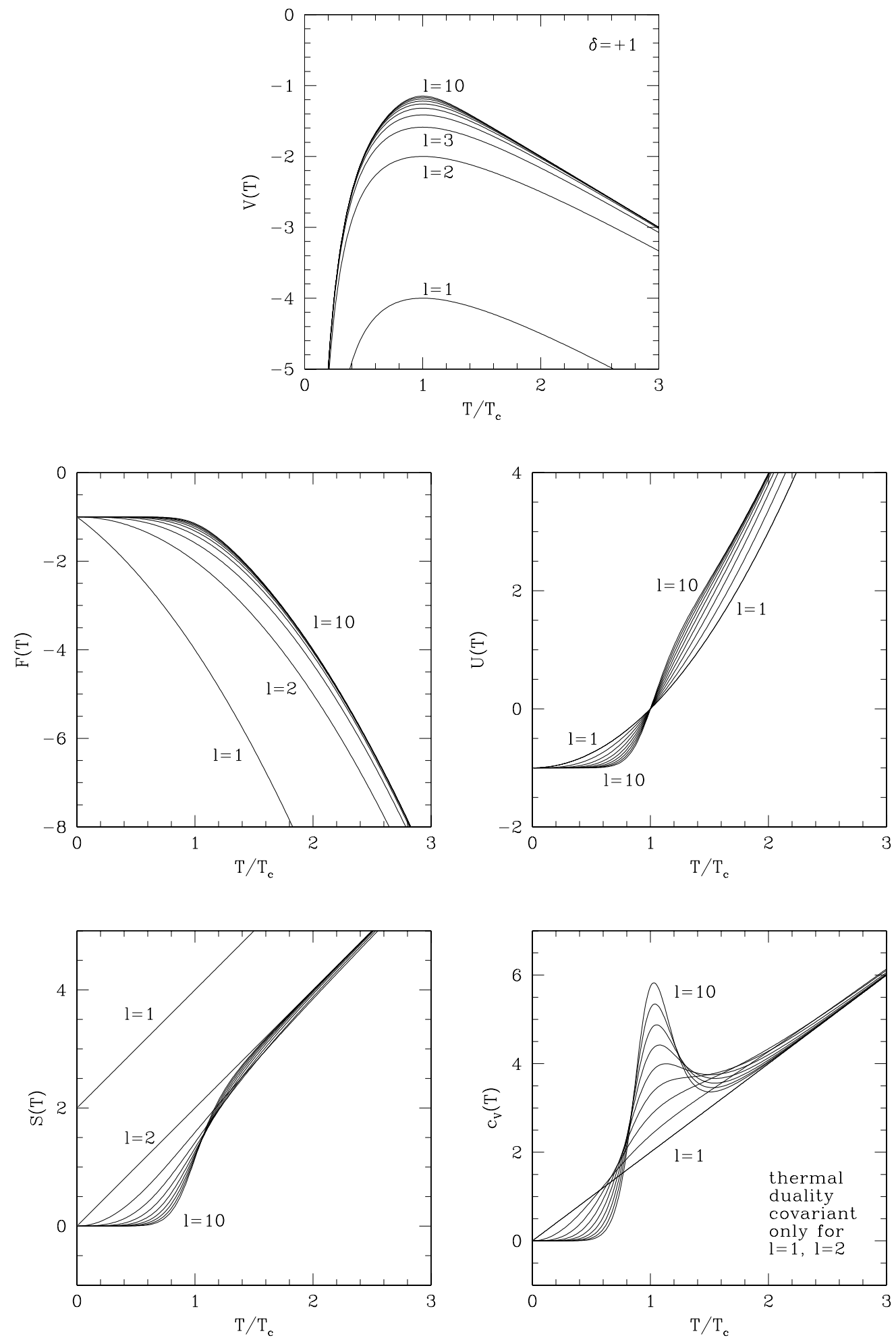

Figure 1: The thermodynamic quantities $\mathcal{V}, F, U, S$, and $c_{V}$ in Eq. (3.11), plotted as functions of $T$ for $1 \leq \ell \leq 10$ and $\delta=+1$, in units of $\mathcal{M} \equiv M_{\text {string }} / 2 \pi=T_{c}$. All quantities except for $c_{V}$ are thermal duality covariant for all $\ell$, while $c_{V}$ is covariant only for $\ell=1,2$. For these values of $\ell$, the entropy and specific heat are exactly linear functions of $T$. Note that $c_{V}$ develops a discontinuity as $\ell \rightarrow \infty$, suggesting the emergence of a second-order phase transition in this limit. 


$$
\begin{aligned}
U^{(1)}(T) & =\left(T^{2}-T_{c}^{2}\right) / T_{c} \\
S^{(1)}(T) & =2\left(T / T_{c}+\delta\right) \\
c_{V}^{(1)}(T) & =2 T / T_{c}
\end{aligned}
$$

where $\delta= \pm 1$. Note that $c_{V}$ has weight $k_{c}=2$ and $\operatorname{sign} \gamma_{c}=1$ for both the $\ell=2$ and $\ell=1$ solutions.

Clearly, the $\ell=1$ and $\ell=2$ solutions are closely related. They share the same expressions for $U$ and $c_{V}$, yet their expressions for $\mathcal{V}, F$, and $S$ are shifted by constants or extra linear terms:

$$
\begin{aligned}
& \mathcal{V}^{(\ell=1)}=\mathcal{V}^{(\ell=2)}-2 \delta \\
& F^{(\ell=1)}=F^{(\ell=2)}-2 \delta T \\
& S^{(\ell=1)}=S^{(\ell=2)}+2 \delta .
\end{aligned}
$$

This shift symmetry will be important in the following.

These $\ell=1,2$ solutions also exhibit other intriguing symmetries. For example, since $F(T) \sim-\left(T^{2}+T_{c}^{2}\right) / T_{c}$ and $U(T) \sim\left(T^{2}-T_{c}^{2}\right) / T_{c}$ for $\ell=2$, we see that $F(i T)=U(T)$ and $U(i T)=F(T)$. In other words, we have the formal symmetry

$$
T \rightarrow i T: \quad F \longleftrightarrow U .
$$

Since $F=T \mathcal{V}$ and $U=-T^{2} d \mathcal{V} / d T$, this immediately leads to a symmetry for $\mathcal{V}(T)$ :

$$
i T \mathcal{V}(i T)=-T^{2} \frac{d \mathcal{V}}{T}
$$

or equivalently

$$
\frac{d \mathcal{V}}{d T}=\frac{\mathcal{V}(i T)}{i T}
$$

This symmetry is remarkable because it relates the troublesome temperature derivative $d \mathcal{V} / d T$ to $\mathcal{V}$ itself. Since $\mathcal{V}(T)$ is defined through a modular integral as in Eq. (2.3), this implies that quantities involving the temperature derivative of $\mathcal{V}$ can now be written as

$$
\frac{d \mathcal{V}}{d T}=-\frac{1}{2} \mathcal{M}^{D-1} \frac{1}{i T} \int_{\mathcal{F}} \frac{d^{2} \tau}{(\operatorname{Im} \tau)^{2}} Z_{\text {string }}(i T)
$$

Moreover, it is easy to show that just as the symmetry (3.16) leads to the symmetry (3.18), it also leads to a symmetry for the second derivative:

$$
\frac{d^{2} \mathcal{V}}{d T^{2}}=\frac{\mathcal{V}(T)}{T^{2}}-\frac{1}{T} \frac{d \mathcal{V}}{d T}=\frac{1}{T^{2}}[\mathcal{V}(T)+i \mathcal{V}(i T)]
$$

It is, in fact, this identity that enforces $S=c_{V}$ for our $\ell=2$ solutions. Similar symmetries also hold for the $\ell>2$ solutions. 


\section{Comparison with explicit one-loop calculations: Temperature dependence of effective potentials}

We now seek to determine the extent to which our closed-form solutions match the results of explicit one-loop modular integrations of the sort that can emerge from actual finite-temperature string ground states. Such comparisons are extremely important because our derivation of the functional forms given in Sect. 3 was "topdown", based entirely on thermal duality symmetries, and did not make reference to any perturbative, order-by-order calculation. Moreover, our discussion was completely model-independent.

Nevertheless, as we shall now discuss, our expressions successfully capture the leading temperature dependence of the one-loop effective potentials for a variety of modular integrals involving time/temperature compactifications on $S^{1}$ (circles) and $S^{1} / Z_{2}$ (orbifolds). Moreover, this will occur for all spacetime dimensions $D \geq 2$. As we shall see, the precision with which this occurs will ultimately lead us to conjecture that our solutions actually represent the exact solutions for the corresponding string effective potentials when results from all orders of perturbation theory (and perhaps even non-perturbative effects) are included.

\subsection{Calculating the one-loop effective potential}

Let us first recall the calculation of the one-loop effective potential for a finitetemperature string ground state in which the time/temperature direction is compactified on a circle. This is appropriate, e.g., for compactifications of the bosonic string, and we shall consider such circle compactifications for most of what follows. In $D$ spacetime dimensions, the one-loop effective potential for such compactifications takes the form in Eq. (2.3), where

$$
Z_{\text {string }}(\tau, T) \equiv Z_{\text {model }}(\tau) Z_{\text {circ }}(\tau, T) \text {. }
$$

Here $Z_{\text {model }}$ represents the trace over the Fock space of states $(i . e$. , the partition function) of the string model in question, formulated at zero temperature. For example, in the case of the bosonic string compactified to $D$ spacetime dimensions, $Z_{\text {model }}$ takes the general form

$$
Z_{\text {model }}=(\operatorname{Im} \tau)^{1-D / 2} \frac{\bar{\Theta}^{26-D} \Theta^{26-D}}{\bar{\eta}^{24} \eta^{24}}
$$

where the numerator $\bar{\Theta}^{26-D} \Theta^{26-D}$ schematically represents a sum over the $2(26-D)$ dimensional compactification lattice for left- and right-movers. Note that in general, $Z_{\text {model }}$ is the quantity which appears in the calculation of the one-loop cosmological constant (vacuum energy density) of the model:

$$
\Lambda \equiv-\frac{1}{2} \mathcal{M}^{D} \int_{\mathcal{F}} \frac{d^{2} \tau}{(\operatorname{Im} \tau)^{2}} Z_{\text {model }} .
$$


By contrast, the remaining factor $Z_{\text {string }}$ represents the sum over Matsubara frequencies. For extended objects such as strings, this includes not only "momentum" Matsubara states but also "winding" Matsubara states. For time/temperature circle compactifications, $Z_{\text {string }}$ is given by*

$$
Z_{\mathrm{circ}}(\tau, T)=\sqrt{\operatorname{Im} \tau} \sum_{m, n \in \mathbb{Z}} \bar{q}^{(m a-n / a)^{2} / 4} q^{(m a+n / a)^{2} / 4}
$$

Here the double sum tallies both the Matsubara momentum and winding states, with $q \equiv \exp (2 \pi i \tau)$ and $a \equiv 2 \pi T / M_{\text {string }}=T / T_{c}$ where $T_{c} \equiv M_{\text {string }} / 2 \pi=\mathcal{M}$. Thus, thermal duality symmetry is nothing but the symmetry $(a \leftrightarrow 1 / a, m \leftrightarrow n)$ in Eq. (4.4).

It is important to emphasize that a factorization of the form given in Eq. (4.1) holds only for the simplest finite-temperature string constructions (such as for the bosonic string). In more realistic setups, simple factorizations such as this are not possible, and one typically has more complicated configurations (see, e.g., Refs. [14, 3, 15, 4, 16] ). In this section, however, we shall confine our attention to this simplest case because it is the situation in which thermal duality is most directly manifest.

\subsection{Asymptotic behavior for low and high temperatures}

Given the form of these partition functions, it is straightforward to deduce the leading behavior in the $T \rightarrow 0$ and $T \rightarrow \infty$ limits, and verify that this behavior matches the corresponding behavior of our solutions in Sect. 3. Taking the $T \rightarrow 0$ limit of $Z_{\text {circ }}$, we find

$$
Z_{\text {circ }} \rightarrow \frac{1}{a} \quad \text { as } a \rightarrow 0
$$

This implies the limiting behavior

$$
\mathcal{V}(T) \sim \frac{\Lambda}{T} \quad \text { as } T / T_{c} \rightarrow 0
$$

where $\Lambda$ is the one-loop cosmological constant in Eq. (4.3). This in turn implies that $F(T) \rightarrow \Lambda$ as $T / T_{c} \rightarrow 0$.

However, this leading behavior for $\mathcal{V}(T)$ and $F(T)$ coincides exactly with the $T \rightarrow$ 0 temperature dependence of the solutions found in Sect. 3 for arbitrary $\ell$. In fact,

\footnotetext{
${ }^{*}$ Since we are defining $Z_{\text {circ }}$ to represent the sum over Matsubara frequencies, we do not include the Dedekind $\eta$-function denominators which would traditionally be required in order to interpret $Z_{\text {circ }}$ as the partition function of a boson compactified on a circle of radius $R_{T} \equiv(2 \pi T)^{-1}$. This does not represent a violation of modular invariance, since the extra factor of $\sqrt{\operatorname{Im} \tau}$ in Eq. (4.4) compensates for their absence. Note that this factor offsets the similar factors in $Z_{\text {model }}$ (just as the summation in $Z_{\text {circ }}$ combines with the lattice sums in $Z_{\text {model }}$ ), thereby effectively reducing by one the dimensionality of the resulting finite-temperature string model compared with the dimensionality of the original string model at zero temperature.
} 
this agreement allows us to go one step further and deduce the overall normalization of our solutions for arbitrary $\ell$ with $\delta=+1$ :

$$
\mathcal{V}^{(\ell)}(T)=\frac{\Lambda}{T_{c}} \frac{\left(T^{\ell}+T_{c}^{\ell}\right)^{2 / \ell}}{T T_{c}} .
$$

We can also consider the opposite, high-temperature limit $T \rightarrow \infty$ in Eq. (4.7), obtaining [4, 6, 7]

$$
\mathcal{V}^{(\ell)}(T) \sim \frac{\Lambda}{T_{c}} \frac{T}{T_{c}} \quad \text { as } T \rightarrow \infty
$$

This implies that $F^{(\ell)}(T) \sim T^{2}$ as $T \rightarrow \infty$, correctly reproducing the celebrated high-temperature behavior which signals the reduced number of degrees of freedom in finite-temperature string theory relative to field theory [4. Note that these correct limiting behaviors are obtained for all values of $\ell$.

Having thus verified that our solutions $\mathcal{V}^{(\ell)}(T)$ in Sect. 3 correctly reproduce the expected, leading $T \rightarrow 0$ and $T \rightarrow \infty$ behaviors for all $\ell$, we now turn to a more detailed study of this scaling behavior as a function of temperature. It turns out that this will enable us to understand the role played by the free parameter $\ell$.

In ordinary quantum field theory, the free energy $F(T)$ at large temperatures typically scales like $T^{D}$ where $D$ is the spacetime dimension. This in turn implies that the entropy $S$ should scale like $T^{D-1}$. However, as already noted above, in string theory we have $F(T) \sim T^{2}$ as $T \rightarrow \infty$, implying that $S(T) \sim T$ as $T \rightarrow \infty$. Thus, string theory behaves asymptotically as though it has an effective dimensionality $D_{\text {eff }}=2$.

Of course, the field-theory limit of string theory is expected to occur for $T \ll T_{c}$. Given this, it is interesting to examine the effective dimensionality (i.e., the effective scaling exponent) of our solutions as a function of temperature. In general, it is easiest to define this effective dimensionality $D_{\text {eff }}(T)$ by considering the entropy: since $S^{(\ell)}(T)$ is a monotonically increasing function of $T$, we can define $D_{\text {eff }}(T)$ as the effective scaling exponent at temperature $T$, setting $S^{(\ell)}(T) \sim T^{D_{\text {eff }}-1}$. We thus have, as a general definition,

$$
D_{\mathrm{eff}} \equiv 1+\frac{d \ln S}{d \ln T}=1+\frac{T}{S} \frac{d S}{d T}=1+\frac{c_{V}}{S}
$$

where the last equality follows from the thermodynamic identity $c_{V}=T d S / d T$.

These results for $D_{\text {eff }}(T)$ are plotted in Fig. 2. As we see, each of our solutions successfully interpolates between $D_{\text {eff }}=\ell$ for $T \ll T_{c}$ and $D_{\text {eff }}=2$ for $T \gg T_{c}$. Indeed, only the $\ell=2$ solution has $D_{\text {eff }}=2$ for all $T$.

Given this observation, it is now possible to interpret our solutions physically. For small temperatures $T \ll T_{c}$, the entropy behaves as we expect on the basis of field theory, growing according to the power-law $S^{(\ell)}(T) \sim T^{\ell-1}$. Indeed, this is nothing but the high-temperature limit of the low-energy effective field theory, which leads us 


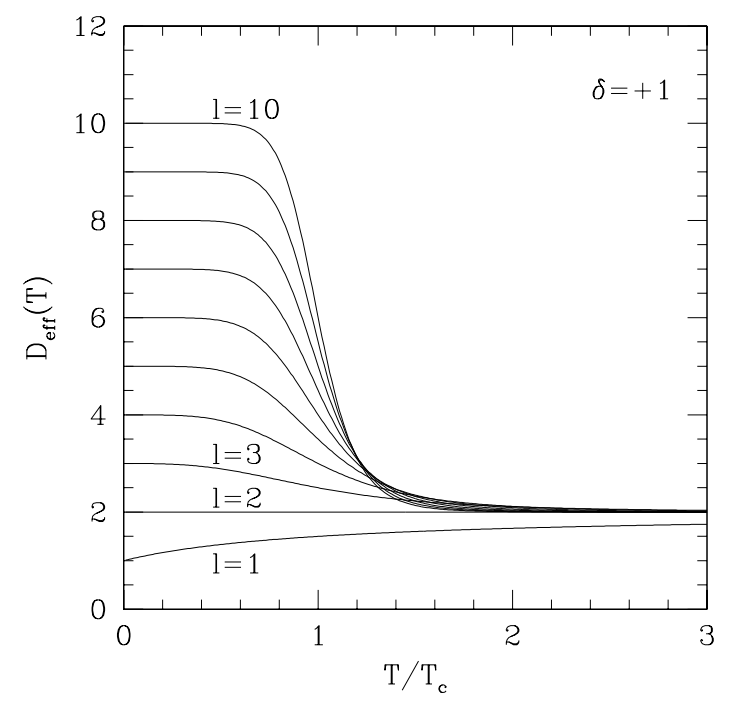

Figure 2: The effective dimensionalities $D_{\text {eff }}$ of our thermodynamic solutions, plotted as functions of $T$ for $1 \leq \ell \leq 10$ and $\delta=+1$. All of our solutions successfully interpolate between $D_{\text {eff }}=\ell$ for $T \ll T_{c}$ and $D_{\text {eff }}=2$ for $T \gg T_{c}$. Only the $\ell=2$ solution has $D_{\text {eff }}=2$ for all $T$.

to interpret $\ell$ as the spacetime dimension $D$. However, as $T$ approaches the reduced string scale $T_{c}$, we see that this asymptotic behavior begins to change, with the $T^{\ell-1}$ growth in the entropy ultimately turning into the expected linear growth for $T \gg T_{c}$. This is then the asymptotic string limit.

Of course, our identification of $\ell$ as the spacetime dimension $D$ is subject to one important caveat. Since $D$ can be defined only through the high-temperature limit of the underlying field theory, our identification of $\ell$ with $D$ assumes that we can properly identify the high-temperature field-theory limit with the low-temperature string-theory limit for which $S^{(\ell)}(T) \sim T^{\ell-1}$. In other words, this identification of $\ell$ with $D$ is sensitive to the manner in which the high-temperature limit of field theory matches onto what ultimately becomes the low-temperature limit of string theory. However, we see from Fig. 2 that in all our solutions, $D_{\text {eff }}$ remains very close to $\ell$ for almost all of the temperature range up to $T_{c}$. Thus, we expect our association of $\ell$ with $D$ to be reasonably accurate. Moreover, in the special case with $\ell=2$, we know that $D_{\text {eff }}=2$ for all $T$. We thus expect that this case should correspond to $D=2$ exactly.

If we consider the same issue from the perspective of the free energy, we can also immediately see the origin of this difference between the high-temperature scaling behaviors in field theory and in string theory. Note that our solution for the free 
energy can be written as

$$
F^{(\ell)}(T)=\Lambda\left[1+\left(\frac{T}{T_{c}}\right)^{\ell}\right]^{2 / \ell}
$$

where we have inserted the normalization factor $\Lambda$ determined above. Expanding this solution for small temperatures, we find

$$
F^{(\ell)}(T) \sim \Lambda+\frac{2 \Lambda}{\ell}\left(\frac{T}{T_{c}}\right)^{\ell}+\ldots \quad \text { for } T \ll T_{c} .
$$

Thus, as already observed above, $F^{(\ell)}(T)$ begins with a constant term $\Lambda$; the fieldtheoretic power-law scaling $T^{\ell}$ appears only at subleading order. However, it is precisely this constant term which ultimately determines the high-temperature scaling behavior in string theory. Recall that if $f$ is a general weight- $k$ covariant function scaling as $f(T) \sim T^{p}$ at small temperatures, then $f$ must scale as $f(T) \sim T^{k-p}$ at high temperatures. Thus, the unusual string-theoretic scaling behavior $F(T) \sim T^{2}$ at high temperatures can ultimately be attributed to the fact that $F(T)$ leads with a constant term $\Lambda$ at small temperatures.

Many of these facts are already well known as general statements in the string literature (see, e.g., Ref. [7]). What we are observing here, however, is that our functional forms correctly exhibit all of these properties simultaneously.

\subsection{Direct comparison for all temperatures}

Since we have already determined that our solutions exhibit the expected low- and high-temperature scaling behaviors for all $\ell$, the question now boils down to whether these solutions correctly match the expected temperature dependence at intermediate temperatures where $T \approx T_{c}$. In other words, we now wish to do a direct comparison at all temperatures.

For simplicity, we begin in $D=2$ by considering model-independent situations in which we set $Z^{\dagger} Z_{\text {model }}$ to 1 . Since $Z_{\text {model }}$ does not contain any temperature dependence of its own, this simplification enables us to focus directly on the temperature dependence arising from $Z_{\text {circ }}$. Our expression for $\mathcal{V}(T)$ from Eqs. (2.3), (4.1), and (4.4) then reduces to

$$
\mathcal{V}^{(D=2)}(T)=-\frac{1}{2} \mathcal{M} \int_{\mathcal{F}} \frac{d^{2} \tau}{(\operatorname{Im} \tau)^{3 / 2}} \sum_{m, n \in \mathbb{Z}} \bar{q}^{(m a-n / a)^{2} / 4} q^{(m a+n / a)^{2} / 4},
$$

with a corresponding "cosmological constant" given by

$$
\Lambda=-\frac{1}{2} \mathcal{M}^{2} \int_{\mathcal{F}} \frac{d^{2} \tau}{(\operatorname{Im} \tau)^{2}}=-\frac{\pi}{6} \mathcal{M}^{2} .
$$

\footnotetext{
${ }^{\dagger}$ Setting $Z_{\text {model }}=1$ does not violate the form given in Eq. (4.2) since we can equivalently write $Z_{\text {model }}=\left|\vartheta_{2} \vartheta_{3} \vartheta_{4}\right|^{16} /\left(2^{16}|\eta|^{48}\right)$ where $\vartheta_{i}$ are the Jacobi theta functions satisfying $\vartheta_{2} \vartheta_{3} \vartheta_{4}=2 \eta^{3}$.
} 
Since $D=2$ in this case, we expect that our expression for $\mathcal{V}^{(D=2)}(T)$ should directly match onto our $\ell=2$ solution. Remarkably, this is exactly what occurs: $\mathcal{V}^{(D=2)}(T)$ is exactly equal to our $\ell=2$ solution $\mathcal{V}^{(\ell=2)}(T)$ with $\delta=+1$ :

$$
\mathcal{V}^{(D=2)}(T)=-\frac{\pi}{6} \frac{T^{2}+T_{c}^{2}}{T}
$$

where we have used the fact that $T_{c}=\mathcal{M}$. Note that Eq. (4.14) holds for all temperatures $T$. Thus, our closed-form $\ell=2$ solution from Sect. 3 exactly reproduces the complete temperature dependence corresponding to the $D=2$ circle compactification in Eq. (4.12)!

Mathematically, this is a rather surprising result. In Eq. (4.12), the temperature dependence of $\mathcal{V}^{(D=2)}(T)$ enters only through the quantity $a \equiv T / T_{c}$ which appears in the exponents of $q$ and $\bar{q}$; this temperature dependence, taking the form of a sum of $\tau$-dependent exponentials, is then integrated over the fundamental domain of the modular group. Nevertheless, we find that the net result of this integration is to produce the simple, closed-form result given in Eq. (4.14). Moreover, as we have already seen in Sect. 3, this temperature dependence is given by precisely the functional form which is necessary in order to ensure that all thermodynamic quantities, including both the entropy and the specific heat, are thermal duality covariant.

This agreement provides an important link between the "top-down" analysis of Sect. 3 and our direct "bottom-up" string calculation. This agreement is especially illuminating, given that our "top-down" derivation made use of a powerful, nonperturbative duality symmetry, while our "bottom-up" string calculation represents only a one-loop result. Taking this agreement seriously, we are tempted to view the one-loop result for this $D=2$ example as "exact", receiving no further contributions at higher loops. Of course, in the absence of an actual string model underlying the expression in Eq. (4.12), this statement is only meant to be suggestive.

Before leaving the $D=2$ special case, we remark that time/temperature circle compactifications are not the only possibility in the construction of finite-temperature string ground states. Another choice (perhaps even a preferred choice phenomenologically [9]) is to compactify on an $S^{1} / \mathbb{Z}_{2}$ orbifold, i.e., a line segment. Indeed, under our factorization assumption in Eq. (4.1), these two choices represent the only two consistent geometries on which a finite-temperature string ground state may be formulated [18. In the case of an orbifold compactification, we simply replace $Z_{\text {circ }}$ in Eq. (4.4) with 18

$$
Z_{\mathrm{orb}}(\tau, T)=\frac{1}{2} Z_{\text {circ }}(\tau, T)+Z_{\text {circ }}\left(\tau, T_{c}\right)-\frac{1}{2} Z_{\text {circ }}\left(\tau, T_{c} / 2\right)
$$

In this expression, the first term represents the contributions from the untwisted states, while the remaining terms are temperature-independent (i.e., they are evaluated at fixed specified temperatures which are independent of $T$ ) and represent the contributions from the twisted states. Since we already know the complete temperature dependence arising from $Z_{\text {circ }}$ in Eq. (4.14), we immediately find that the 
effective potential in the orbifold case has the exact closed-form solution

$$
\mathcal{V}_{\mathrm{orb}}^{(D=2)}=-\frac{\pi}{12}\left[\frac{T^{2}+T_{c}^{2}}{T}+\frac{3}{2}\right] .
$$

Of course, this is nothing but our $Z_{\text {circ }}$ solution, rescaled and shifted by an additive constant. However, recall from Eq. (3.15) that the $\ell=1$ solution differs from the $\ell=2$ solution merely through such an additive shift. Since the circle solution corresponds to $\ell=2$, this suggests that our orbifold solution in Eq. (4.16) can be expressed exactly as a linear combination of the $\ell=2$ and $\ell=1$ solutions in Eq. (4.7), and this is indeed the case:

$$
\mathcal{V}_{\text {orb }}^{(D=2)}=\frac{3}{4} \mathcal{V}^{(\ell=1)}+\frac{1}{4} \mathcal{V}^{(\ell=2)}
$$

where we have taken $\delta=+1$ in the $\ell=1$ solution. Once again, we stress that this is an exact representation for the complete temperature dependence of the $D=2$ orbifold case. Note that in writing this expression, we have identified the normalization constant $\Lambda=\Lambda_{\text {circ }} / 2$; this follows from the low-temperature limit of Eq. (4.15), even though this $\Lambda$ is no longer the cosmological constant of the original zero-temperature model. Also note that even though the $\ell=1,2$ solutions are shifted relative to each other by an additive constant, we cannot write $\mathcal{V}_{\text {orb }}$ purely in terms of either of the $\ell=1$ solutions (with $\delta= \pm 1$ ) because the additive shifts in these $\ell=1$ solutions are \pm 2 relative to the $\ell=2$ solution. According to Eq. (4.16), however, our shift constant is $3 / 2$ relative to the $\ell=2$ solution. This fact has some important consequences which we shall discuss in Sect. 6 .

Given the exact orbifold solution in Eq. (4.16), we can immediately see the thermodynamic effects of compactifying the time/temperature dimension on an orbifold rather than a circle. While the internal energy and specific heat are unaffected by this choice, the free energy picks up an additional linear term and the entropy picks up an additive constant. The latter has been called a "fixed-point" entropy [9] since it arises from the fixed points of the $S^{1} / \mathbb{Z}_{2}$ orbifold and survives even in the $T \rightarrow 0$ limit; in the present case this fixed-point entropy is given exactly as

$$
S_{\text {fixed-point }}=\pi / 8 \text {. }
$$

Let us now proceed to consider the case in higher dimensions $D>2$. As might be expected, things are more complicated. For arbitrary $D$, the expression in Eq. (4.12) now generalizes to

$$
\mathcal{V}^{(D)}(T)=-\frac{1}{2} \mathcal{M}^{D-1} \int_{\mathcal{F}} \frac{d^{2} \tau}{(\operatorname{Im} \tau)^{(D+1) / 2}} \sum_{m, n \in \mathbb{Z}} \bar{q}^{(m a-n / a)^{2} / 4} q^{(m a+n / a)^{2} / 4}
$$

where we incorporate the $D$-dependent prefactor $(\operatorname{Im} \tau)^{1-D / 2}$ from $Z_{\text {model }}$ but continue to disregard the rest of this function for simplicity. While Eq. (4.19) is not modular invariant, it captures the dominant $T$ - and $D$-dependence that we wish to explore. 
As evident from Eq. (4.19), the net effect of altering the spacetime dimension is to change the power of the $(\operatorname{Im} \tau)$ factor that appears in the measure of the integral. If we view the $Z_{\text {circ }}$ integrand as a power series in $q$ and $\bar{q}$, with each term separately integrated and then summed to produce the effective potential $\mathcal{V}^{(D)}$, we see that the dominant effect of changing the spacetime dimension is to reweight the contributions from each term in the $Z_{\text {circ }}$ power series because they are now being integrating over the modular-group fundamental domain with an altered measure. Thus, it is not immediately apparent how the temperature dependence found in the $\ell=2$ case should change.

Nevertheless, we find that our functions $\mathcal{V}^{(\ell)}(T)$ from Sect. 3 continue to successfully capture the dominant temperature dependence of the resulting integrals, with $\ell=D$. Unlike the case with $D=\ell=2$, this agreement is only approximate rather than exact. Nevertheless, we find that this agreement holds to within one or two percent over the entire temperature range $0 \leq T \leq \infty$. Indeed, if we were to superimpose a plot of $\mathcal{V}^{(D)}(T)$ over the plot of $\mathcal{V}^{(\ell)}(T)$ in Fig. 1 taking $\ell=D$, we would not be able to discern the difference at the level of magnification in Fig. 1]

Once again, this is a rather striking result, suggesting that our functional forms continue to capture the dominant temperature dependence, even in higher dimensions. Of course, for $D>2$, our solutions and the above one-loop results do not agree exactly. However, given the significant role played by thermal duality in constraining the form of the effective potential to the specific functional forms that we have found in Sect. 3, and given the precision with which the above one-loop results appear to match these functional forms, it is natural to attribute the failure to obtain an exact agreement for $D>2$ to the fact that $\mathcal{V}^{(D)}(T)$ in Eq. (4.19) is itself only a one-loop approximation. We thus are led to conjecture that our functional forms $\mathcal{V}^{(\ell)}(T)$ indeed represent the exact solutions for the finite-temperature effective potentials, even in higher dimensions, and that these solutions will emerge only when the contributions from all orders in perturbation theory are included. Viewed from this perspective, it is perhaps all the more remarkable that we found an exact agreement for $D=2$, suggesting that the one-loop result is already exact in this special case, with no further renormalization.

Let us now consider what happens if we do not make the simplification that $Z_{\text {model }}=1\left[\right.$ or $\left.Z_{\text {model }}=(\operatorname{Im} \tau)^{1-D / 2}\right]$. Of course, in order to select an appropriate $Z_{\text {model }}$, we must actually construct a bona-fide string model (e.g., a specific bosonic string compactification); moreover, this model must be tachyon-free if our effective potential is to be finite. These constraints force $Z_{\text {model }}$ to take the form $Z \sim 1+\sum_{m n} a_{m n} \bar{q}^{m} q^{n}$ where $a_{m n}=0$ if $m=n<0$ (no physical tachyons). The presence of the leading constant term in the power expansion means that the leading temperature dependence of $\mathcal{V}^{(D)}$ will continue to be the same as we had when we merely set $Z_{\text {model }}=1$. Indeed, the contributions from the higher terms in $Z_{\text {model }}$ are exponentially suppressed relative to the leading term, which means that the net effect of the extra, model-dependent terms in $Z_{\text {model }}$ is to provide an exponentially sup- 
pressed reweighting of the contributions from the different terms in the power-series expansion of $Z_{\text {circ }}$. Thus, the net effect of inserting a non-trivial $Z_{\text {model }}$ into $\mathcal{V}^{(D)}$ is merely to change the subleading temperature dependence in a model-dependent way. Thus, we conclude that the leading temperature dependence continues to be captured by our solutions $\mathcal{V}^{(\ell)}(T)$ even when $Z_{\text {model }} \neq 1$; indeed, this is the universal, modelindependent contribution. Moreover, if our conjecture is correct, then we expect these subleading model-dependent contributions to be washed out as higher-order contributions are included in the perturbation sum. Just as for the $D=2$ special case, similar remarks apply if we replace the thermal compactification geometry from a circle to an orbifold.

Finally, let us briefly comment on the most general cases of all, namely those in which the finite-temperature partition functions do not factorize as in Eq. (4.1). Such cases include compactifications with temperature-dependent Wilson lines, and are expected to emerge in heterotic or Type II theories where non-trivial phases must be introduced in the combined thermal partition function (ultimately due to presence of spacetime fermions). For example, non-factorized thermal partition functions emerge for finite-temperature string theories whose zero-temperature limits are spacetime supersymmetric; these theories necessarily have thermal partition functions in which the cancellations inherent in supersymmetry are non-trivially mixed with the Matsubara sums (see, e.g., the examples in Refs. [14, 3, 15, 4, 16]). In such cases, however, the effective potentials do not generally exhibit thermal duality indeed, such theories may be considered to be finite-temperature string ground states in which thermal duality is spontaneously broken. Such theories are therefore beyond the scope of this paper. We shall, however, present an analysis of such theories in Ref. 19, where we will show that an analogue of this bootstrap approach can be developed for such theories as well.

\section{Effective scaling dimensionalities: Connection to holography?}

In Eq. (4.9), we defined the notion of an effective dimensionality $D_{\text {eff }}$ which governs the scaling behavior of the entropy $S(T)$, with $S(T) \sim T^{D_{\text {eff }}-1}$. As we have seen, this scaling coefficient generally ranges from $D_{\text {eff }}=D$ as $T \rightarrow 0$ to $D_{\text {eff }}=2$ as $T \rightarrow \infty$. The limiting behavior as $T \rightarrow 0$ is precisely as expected on the basis of ordinary quantum field theory, while the opposite limiting behavior as $T \rightarrow \infty$ is precisely as required by thermal duality.

This reduction in the effective dimensionality of the system at high temperatures is extremely reminiscent of holography (such an interpretation can also be found, e.g., in Refs. 9, 17]). Indeed, the scaling of our thermodynamic quantities departs from the ordinary $D$-dimensional scaling that would be expected on the basis of quantum field theory, and begins to behave as though the number of accessible degrees 
of freedom populates not the full $D$-dimensional spacetime, but rather a subspace of smaller dimensionality. Of course, an analysis formulated in flat space (such as ours) cannot address questions pertaining to the geometry of this subspace, and thus cannot determine whether the surviving degrees of freedom are really to be associated with a subspace or boundary of the original geometry. However, from the restricted perspective emerging from a mere counting of states, we see our scaling behavior differs significantly from field-theoretic expectations, suggesting some sort of reduction in the effective dimensionality associated with thermally accessible degrees of freedom as $T \rightarrow \infty$.

Of course, taking the $T \rightarrow \infty$ limit is merely of formal interest. In a theory with thermal duality, there is no difference between the range $T>T_{c}$ and the range $T<T_{c}$ since these ranges capture the same physics and are thus indistinguishable. Or, phrased another way, thermal duality tells us that there is a "maximum" temperature in the same sense that T-duality tells us there is a minimum radius. This is also consistent with our expectation that there should be a Hagedorn-type phase transition at or near $T_{c}$, with the theory ultimately entering a new phase marked by new degrees of freedom. Thus, we should really only consider the range $0 \leq T \leq T_{c}$.

Given this, let us consider the value of $D_{\text {eff }}$ not as $T \rightarrow 0$ or $T \rightarrow \infty$, but as $T \rightarrow T_{c}$. As discussed above, this is truly the "high-temperature" limit of string theory. With our specific closed-form solutions $\mathcal{V}^{(\ell)}(T)$ in Eq. (3.11), the general definition in Eq. (4.9) yields

$$
D_{\text {eff }}(T)=\frac{2 T^{D}+D T_{c}^{D}}{T^{D}+T_{c}^{D}}
$$

where we have identified $\ell=D$. We thus obtain

$$
D_{\text {eff }}\left(T_{c}\right)=\frac{1}{2}(2+D) .
$$

This result indicates that $D_{\text {eff }}\left(T_{c}\right)<D$ for all $D>2$. In other words, for all $D>2$, the effective scaling of the number of degrees of freedom at high temperatures is reduced compared with our field-theoretic expectations at low temperatures. However, taking the predictions of holography seriously, we can ask when this reduction in $D_{\text {eff }}$ is truly "holographic" in the sense that $D_{\text {eff }}$ is reduced by exactly one unit as $T \rightarrow T_{c}$, dropping from $D$ to $D-1$. This would be analogous, for example, to what occurs for black holes, where quantities such as entropy scale not with the three-volume of the black hole, but with its area. Remarkably, demanding that $D_{\text {eff }}$ drop by precisely one unit yields

$$
D_{\text {eff }}\left(T_{c}\right)=D-1 \quad \Longrightarrow \quad D=4 .
$$

Thus, we see that it is precisely in four dimensions that our solutions behave "holographically" in the range $0 \leq T \leq T_{c}$, with the effective scaling dimensionality falling exactly by one unit from $D_{\text {eff }}=4$ to $D_{\text {eff }}=3$. This behavior is plotted in Fig. 3 , 


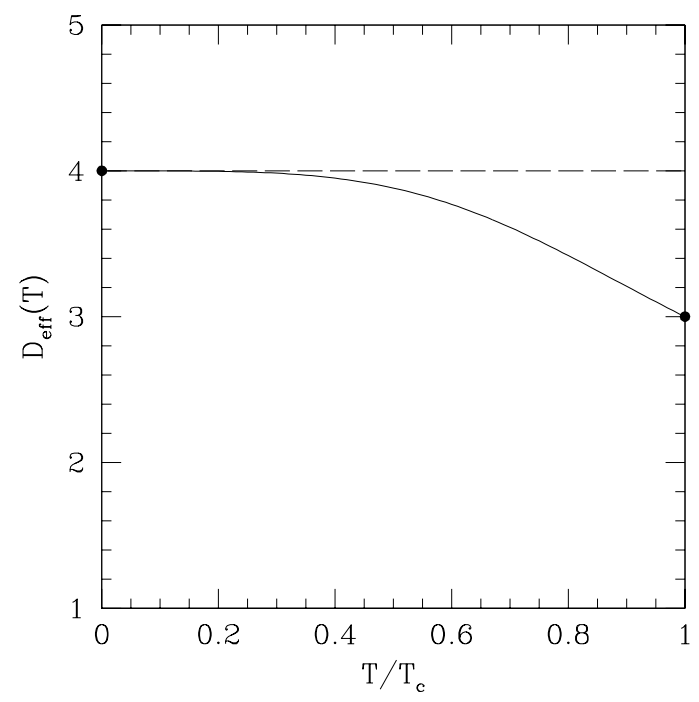

Figure 3: The effective dimensionality $D_{\text {eff }}$ of our four-dimensional thermodynamic solutions, plotted as a function of $T$. These solutions behave "holographically" in the range $0 \leq T \leq T_{c}$, with the effective scaling dimensionality falling exactly from $D_{\text {eff }}=4$ to $D_{\text {eff }}=3$. The dotted line indicates the behavior that would be expected within quantum field theory.

While it is tempting to interpret this reduction in $D_{\text {eff }}$ as a holographic effect, we again caution that our setup (based on a flat-space calculation) is incapable of yielding the additional geometric information that this claim would require. Such an analysis is beyond the scope of this paper, and would require reformulating the predictions of thermal duality for string theories in non-trivial $D$-dimensional backgrounds, and then determining whether we could formulate a map between degrees of freedom in the bulk of the $D$-dimensional volume and those on the $(D-1)$-dimensional boundary of this volume. Nevertheless, we find this reduction in $D_{\text {eff }}$ to be an extremely intriguing phenomenon, especially since our exact solutions lead to a strictly "holographic" reduction in $D_{\text {eff }}$ for $D=4$. We thus believe that this approach towards understanding the relation between thermal duality and holography is worthy of further investigation.

\section{Discussion}

In this paper, we set out to address a very simple issue: even though thermal duality is an apparent fundamental property of string theory, emerging as a consequence of Lorentz invariance and T-duality, the rules of classical thermodynamics do not appear to respect this symmetry. Even when the vacuum amplitude $\mathcal{V}(T)$ exhibits thermal duality, thermodynamic quantities such as entropy and specific heat 
do not. Given this situation, we sought to determine whether special string ground states might exist such that thermal duality will nevertheless be exhibited by all of the usual thermodynamic quantities of interest.

We began by deriving specific solutions $\mathcal{V}(T)$ such that thermal duality is preserved not only for the free and internal energies, but also for the entropy and specific heat. The complete set of such solutions is itemized in Eqs. (3.11), (3.13), and (3.14). While the solutions for general $\ell$ preserve thermal duality for all thermodynamic quantities except the specific heat, the $\ell=1,2$ solutions preserve thermal duality for all of the thermodynamic quantities.

We then investigated the extent to which these solutions might emerge from modular integrals of the sort that would be expected in one-loop calculations from actual string ground states. Remarkably, we found that our $\ell=1,2$ closed-form solutions provide exact representations for $D=2$ modular integrals corresponding to time/temperature compactifications on circles and orbifolds. This agreement is particularly encouraging from a mathematical standpoint, since our derivation of these functional forms is entirely "top-down", proceeding only from thermal duality symmetry principles, and has nothing to do with specific "bottom-up" constructions involving specific one-loop modular integrations. The fact that these two approaches agree exactly, yielding the same results even in highly simplified cases, suggests that thermal duality is likely to play an important role governing self-consistent string ground states. Indeed, as we saw in Sect. 5 , these $\ell=1,2$ solutions also ensure that modular invariance is also preserved for all relevant thermodynamic quantities.

By contrast, our remaining $\ell>2$ closed-form solutions do not serve as exact representations of appropriate $D>2$ modular integrals. Nevertheless, we found that they provide extremely accurate approximations to such integrals in a wide variety of cases. This led us to conjecture that our $\ell>2$ functional forms may indeed provide exact solutions for the effective potentials corresponding to wide classes of finite-temperature string ground states once the contributions from all orders of perturbation theory (and perhaps even non-perturbative effects) are included. After all, our method of deriving these solutions rests solely on the requirement of thermal duality, a symmetry which (like the T-duality from which it is derived) holds to all orders in perturbation theory, and even non-perturbatively. Thus, if this conjecture is correct, it is perhaps not surprising that our $\ell>2$ solutions transcend the results of intrinsically one-loop calculations.

In this connection, it is important to stress that the free energy $F(T)$ exhibits thermal duality order by order in string theory. Our conjecture does not alter this behavior. What we are conjecturing, however, is that the sum of these order-by-order perturbative functions $F(T)$ actually exhibits an additional symmetry, one which guarantees that the entropy $S(T)$ is also duality covariant. Thus, while thermal duality is indeed preserved order by order for the string free energy, we are conjecturing that the entropy, which normally fails to exhibit this symmetry at any order, actually will exhibit this symmetry when all of these separate order-by-order contributions are 
summed together.

Of course, this conjecture requires not only a special temperature behavior at each order, but also a specific value of the string coupling $\kappa$. To see this, recall that the full free energy $F(\kappa, T)$ depends not only on the temperature $T$ but also on the string coupling $\kappa$. Specifically, if $F_{g}(T)$ is the genus- $g$ contribution to the total free energy $F(\kappa, T)$, then

$$
F(\kappa, T)=\sum_{g=1}^{\infty} \kappa^{2(g-1)} F_{g}(T) .
$$

In general, the genus- $g$ free energy transforms as a weight- $2 g$ duality-covariant function,

$$
F_{g}\left(T_{c}^{2} / T\right)=\left(T_{c} / T\right)^{2 g} F_{g}(T),
$$

which is why the total free energy, like its genus-one contribution, transforms as a weight-two duality-covariant function:

$$
F\left(\kappa, T_{c}^{2} / T\right)=\left(T_{c} / T\right)^{2} F\left(\kappa T_{c} / T, T\right) .
$$

The corresponding shift in the string coupling is precisely analogous to what occurs in T-duality. However, since the string coupling $\kappa$ parametrizes the relative weightings of the contributions from each genus, any new symmetry which appears only in the sum over all genera must hold only for a specific value of the string coupling. Our conjecture, which claims that the full free energy $F(\kappa, T)$ must have the exact temperature dependence given by $F^{(\ell)}(T)$ with $\ell=D$, must therefore hold only for a specific value of the string coupling which in turn must presumably be fixed by other, non-perturbative effects.

While these are exciting speculations, we are nevertheless left with our original question as to whether there exist special finite-temperature string ground states for which all relevant thermodynamic quantities exhibit thermal duality. For $D>2$, it seems that such states do not exist: even if the above conjecture is correct and the exact effective potentials of such string models match our $\ell>2$ functional forms, these functional forms do not preserve thermal duality covariance for the specific heat. Only the $\ell=1,2$ solutions have this property. However, for $D=2$, the answer to this question may be somewhat more positive, for the case of time/temperature circle compactifications with $Z_{\text {model }}=1$ appears to yield exactly what we require. Thus, even when we take $Z_{\text {model }} \neq 1$, our above conjecture suggests that the corrections that are induced by the non-trivial $Z_{\text {model }}$ might ultimately disappear when contributions from all orders are included. Indeed, in this way, our conjecture would lead to a model-independent universal form for the effective potentials corresponding to such compactifications. However, it is important to realize that even if the circle case leads to a duality-covariant entropy and specific heat, the corresponding orbifold case certainly does not. Since the additive shift in the effective potential that accrues in passing from the circle to the orbifold is given by $3 / 2$ rather than $\delta= \pm 1$, the orbifold case corresponds not to the $\ell=1$ solution but rather to a linear combination 
of the $\ell=1$ and $\ell=2$ solutions, as indicated in Eq. (4.17). The resulting entropy is thus a linear combination of two terms with different duality weights, and fails to be covariant at all. Of course, the specific heat continues to be covariant, since the specific heat is unaffected by the contributions from the orbifold fixed points.

What then are we to conclude from this analysis? Clearly, if string theory is to resurrect thermal duality for quantities such as entropy and specific heat, the miracle is not likely to lie in the clever choice of a string ground state. Rather, the miracle is more likely to lie in the structure of thermodynamics itself, as a possible string-theoretic modification of the usual rules of classical thermodynamics according to which quantities such as entropy and specific heat are calculated. Indeed, as we shall see in Ref. [20], such an approach is capable of restoring thermal duality to all thermodynamic quantities — regardless of the specific ground state - and leads to a new, manifestly duality-covariant string thermodynamics. The development of such a theory will be explored in Ref. [20].

\section{Acknowledgments}

This work is supported in part by the National Science Foundation under Grants PHY-0071054 and PHY-0301998, and by a Research Innovation Award from Research Corporation. We wish to thank D. Marolf, R. Myers, R. Roiban, C. Stafford, and G. Torrieri for discussions. 


\section{References}

[1] K. R. Dienes and M. Lennek, Thermal Duality Confronts Entropy: A New Approach to String Thermodynamics?, arXiv:hep-th/0312173.

[2] K. H. O'Brien and C. I. Tan, Phys. Rev. D 36 (1987) 1184.

[3] E. Alvarez and M. A. R. Osorio, Nucl. Phys. B 304 (1988) 327 [Erratum-ibid. B 309 (1988) 220].

[4] J. J. Atick and E. Witten, Nucl. Phys. B 310 (1988) 291.

[5] E. Alvarez and M. A. R. Osorio, Phys. Rev. D 40 (1989) 1150.

[6] M. A. R. Osorio, Int. J. Mod. Phys. A 7 (1992) 4275.

[7] For an introduction, see J. Polchinski, String Theory, Vol. I (Cambridge University Press, 1998), Chap. 9.

[8] J. Polchinski, Commun. Math. Phys. 104 (1986) 37.

[9] S. Chaudhuri, Phys. Rev. D 65 (2002) 066008 arXiv:hep-th/0105110.

[10] N. Sakai and I. Senda, Prog. Theor. Phys. 75 (1986) 692 [Erratum-ibid. 77 (1987) 773].

[11] V. P. Nair, A. D. Shapere, A. Strominger and F. Wilczek, Nucl. Phys. B 287 (1987) 402.

[12] B. Sathiapalan, Phys. Rev. Lett. 58 (1987) 1597.

[13] B. McClain and B. D. B. Roth, Commun. Math. Phys. 111 (1987) 539.

[14] R. Rohm, Nucl. Phys. B 237 (1984) 553;

H. Itoyama and T. R. Taylor, Phys. Lett. B 186 (1987) 129;

P. Ginsparg and C. Vafa, Nucl. Phys. B 289 (1987) 414;

J. D. Blum and K. R. Dienes, Phys. Lett. B 414 (1997) 260 arXiv:hep-th/9707148; Nucl. Phys. B 516 (1998) 83 arXiv:hep-th/9707160; Nucl. Phys. B 520 (1998) 93 arXiv:hep-th/9708016.

[15] M. McGuigan, Phys. Rev. D 38 (1988) 552.

[16] C. Kounnas and B. Rostand, Nucl. Phys. B 341 (1990) 641.

[17] S. Chaudhuri, arXiv:hep-th/0208112 
[18] P. Ginsparg, Nucl. Phys. B 295 (1988) 153;

P. Ginsparg, in Proceedings of Les Houches, Session XLIX, 1998 (Elsevier Science Publishers, 1989), edited by E. Brézin and J. Zinn-Justin.

[19] K. R. Dienes and M. Lennek, in preparation.

[20] K. R. Dienes and M. Lennek, Adventures in Thermal Duality (II): Towards a Duality-Covariant String Thermodynamics, arXiv:hep-th/0312217. 